\title{
Peut-on parler de partenariat apprenant en éducation à l'environnement?
}

Yannick Bruxelle

\section{(2) OpenEdition \\ Journals}

Édition électronique

URL : http://journals.openedition.org/ere/6566

DOI : $10.4000 /$ ere.6566

ISSN : 2561-2271

Éditeur

Centr'ERE

Référence électronique

Yannick Bruxelle, « Peut-on parler de partenariat apprenant en éducation à l'environnement? », Éducation relative à l'environnement [En ligne], Volume 3 | 2002, mis en ligne le 20 mars 2002, consulté le 16 avril 2021. URL : http://journals.openedition.org/ere/6566; DOI : https://doi.org/10.4000/ere. 6566

Ce document a été généré automatiquement le 16 avril 2021. 


\title{
Peut-on parler de partenariat apprenant en éducation à l'environnement?
}

\author{
Yannick Bruxelle
}

1 L'explosion du partenariat depuis une vingtaine d'années tant en terme d'affichages de plus en plus clairs et nombreux par les institutions publiques, les collectivités ou les associations, qu'en terme de pratiques pour un nombre croissant de professionnels m'a amenée à m'interroger sur cette nouvelle forme très structurée d'organisation collective.

2 Étant membre de l'éducation nationale, je travaille régulièrement avec des personnes d'autres institutions ou associations afin d'initier et de développer ensemble des projets de partenariats liés à l'éducation à l'environnement (EE) et notamment dans le domaine de la formation continue. Mon intérêt pour cette organisation et ces pratiques m'a amenée à mener pendant trois années une recherche-action en situation professionnelle avec l'Université François Rabelais de Tours et à entraîner les autres formateurs dans cette démarche réflexive.

3 Ainsi, dans le cadre de nos formations de formateurs en éducation à l'environnement, pressentions-nous que le partenariat, au-delà de son efficacité, au-delà du plaisir de travailler ensemble, nous permettait d'apprendre ensemble. Les travaux des deux chercheurs japonais Nonaka et Takeuchi (1997) développant la notion d'entreprises apprenantes et les conditions nécessaires à cette apprenante, m'amenèrent à développer l'hypothèse que le partenariat lui aussi pouvait être une organisation apprenante et à parler de "partenariat apprenant ".

Il s'agissait également pour nous de comprendre pourquoi ces pratiques se développaient de façon quasi systématique dans le domaine de l'éducation à l'environnement, de savoir s'il y avait une cohérence entre ces pratiques partenariales apprenantes et les valeurs de l'éducation à l'environnement et si à terme il était possible d'espérer qu'elles soient un instrument vers un changement social (ce dernier aspect ne sera pas développé ici). 
5 Cet article présentera dans un premier temps l'ensemble de la démarche de recherche depuis l'origine de l'hypothèse du partenariat apprenant jusqu'à la récolte des données, puis il abordera l'interprétation croisée des résultats et leur confrontation aux travaux de Nonaka et Takeuchi (1997). Une troisième partie explorera les indicateurs de qualité du partenariat apprenant et la recherche des cohérences avec l'éducation à l'environnement; enfin la dernière partie cherchera à cerner les atouts et limites de cette notion de partenariat apprenant.

\section{De l'hypothèse du partenariat apprenant à la récolte des données de la recherche}

\section{L'origine de l'hypothèse du partenariat apprenant}

6 Après avoir travaillé les différentes facettes et interprétations du concept de partenariat pouvant aller de l'information mutuelle à la fusion totale en passant par la consultation, la concertation, la coopération et le partenariat de réciprocité, il m'a semblé que, dans le contexte qui était le nôtre, il était important d'y associer l'idée d'une certaine évolution du partenariat dans le temps liée au fait qu'en situation partenariale, on apprend ensemble.

7 J'ai donc approché le concept «savant» d'apprenante, encore absent de nos dictionnaires, non accueilli par la société civile et ne faisant pas non plus l'unanimité dans le monde universitaire.

8 Pourtant il m'a semblé qu'indiquant la durée et renforçant l'idée d'action, d'entrer dans un processus dynamique, il était plus approprié que celui d'apprentissage. Ainsi, " l'apprenante " ne concerne pas des savoirs préexistants qui sont transmis mais des savoirs construits et adaptés à la situation vécue. L'apprenante est donc liée à la production de connaissances par les personnes qui apprennent et elle se situe davantage dans le domaine personnel de «l'être» que dans celui de «l'avoir» (posséder beaucoup de connaissances).

9 Certaines lectures, notamment les ouvrages sous la direction de Jeanne Mallet (1996), m'ont permis de voir que de nombreux auteurs, au-delà de l'apprenante individuelle, parlaient $d$ '« organisations apprenantes "; puis ayant ensuite été très frappée à la lecture de l'ouvrage d'Ikujiro Nonaka et Hirotaka Takeuchi (1997) par leur formulation d'« entreprises apprenantes » et par les étapes de conversion de connaissances qu'ils décrivaient, j'ai été amenée à formuler l'hypothèse suivante: "Le partenariat en éducation à l'environnement est apprenant: il permet d'accéder à des compétences nouvelles. Fondé sur de nouvelles valeurs, il modifie les relations humaines et contribue à des changements sociaux importants ».

10 Ainsi, dans leur ouvrage «La connaissance créatrice : la dynamique de l'entreprise apprenante ", Nonaka et Takeuchi (1997) étudient différentes entreprises japonaises. Ils développent leur théorie de la création de connaissances sous la forme d'une spirale s'appuyant sur ce que vivent les personnes lorsqu'elles font interagir leurs connaissances tacites (les connaissances personnelles, incrustées dans les expériences individuelles et difficiles à articuler au moyen du langage formel) et leurs connaissances explicites (celles facilement exprimées en langage formel donc aisément communicables). Ainsi, selon ces auteurs: «L'organisation ne peut créer de 
connaissances par elle-même sans l'initiative des individus et l'interaction qui a lieu au sein du groupe » (p. 31) et, « si la connaissance n'est pas partagée avec d'autres ou n'est pas amplifiée au niveau du groupe, elle ne permet pas de réaliser un processus en spirale au plan organisationnel. Ce processus en spirale, qui s'étend aux différents niveaux ontologiques, est une des clés pour comprendre la création de connaissances organisationnelles » (p. 245).

Quatre modes de conversion de connaissances sont ainsi définis par Nonaka et Takeuchi (1997) comme le montre la figure 1 ci-après :

- la «socialisation », qui est un processus de partage d'expériences partant de connaissances tacites et produisant de la connaissance dite « sympathique » ou " assimilée »;

- l'« extériorisation », qui articule et convertit les connaissances tacites en concepts explicites ; déclenchée par le dialogue et la réflexion collective, elle produit de la connaissance dite « conceptuelle »;

- la « combinaison", qui configure différents corps de connaissances explicites en les triant, les additionnant, les combinant, les catégorisant et produit de la connaissance dite " systémique »;

- l'« intériorisation", qui est un processus d'incorporation de la connaissance explicite en connaissance tacite produisant une connaissance dite « opérationnelle ».

L'idée était donc de voir, dans la mesure où le partenariat est une forme d'organisation, s'il était possible de transposer les travaux de Nonaka et Takeuchi (1997) dans un contexte tout autre, fort éloigné du contexte de la compétition économique des entreprises, mais basé sur ce que j'ai appelé le «partenariat apprenant » et ce, dans le contexte éducatif qui était le nôtre.

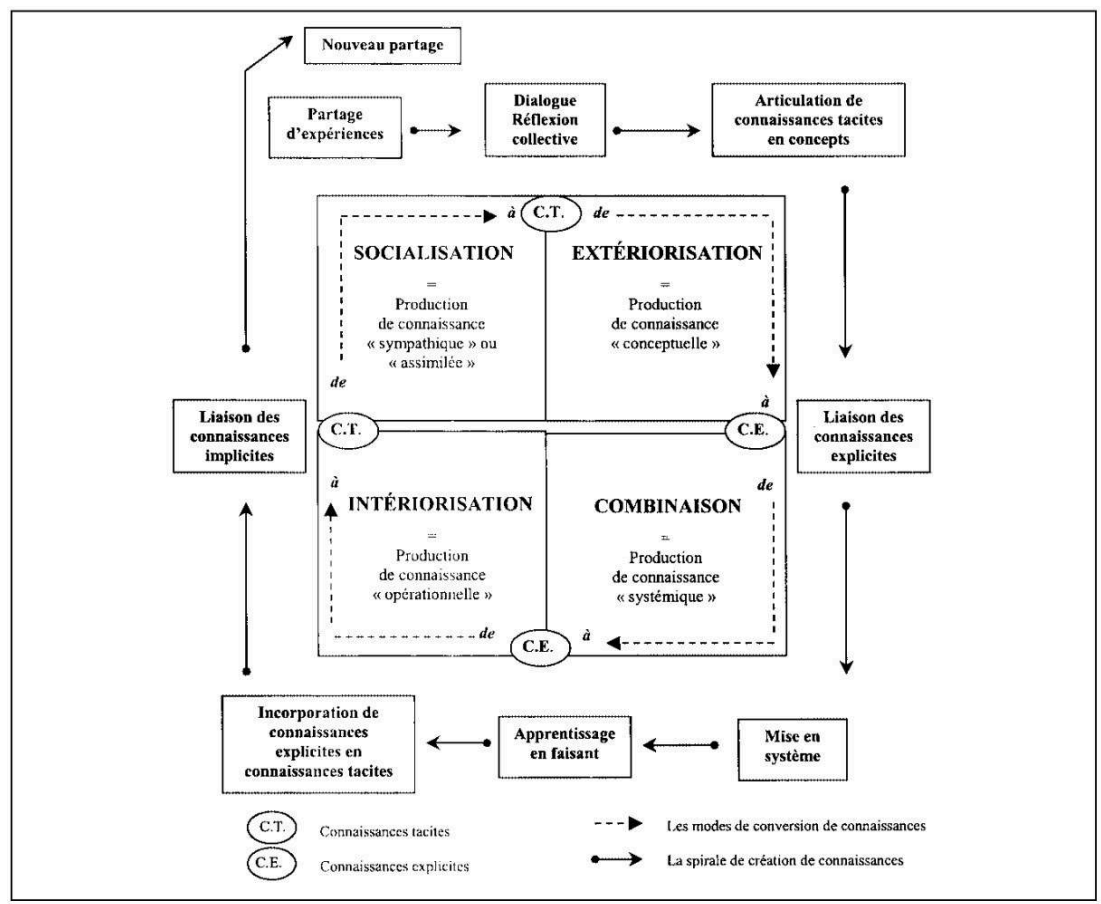

Figure 1 : La création de connaissances

(adaptée de Nonaka et Takeuchi (1997) à partir des schémas des auteurs p. 83, 93 et 94) 


\section{Un contexte partenarial de recherche}

13 Notre travail s'appuie sur des actions de formation de formateurs pendant la durée d'une année scolaire, inscrites à la fois au plan national de formation de l'éducation nationale française et donc destinées à des formateurs d'enseignants venant de toute la France, mais aussi au programme de l'Institut de formation et de recherche en éducation à l'environnement (Ifrée-ORE) et donc ouvertes à d'autres formateurs du monde des associations ou des collectivités.

14 Au-delà du mélange des stagiaires, l'organisation et l'animation du stage réunissaient quatre partenaires du Poitou-Charentes, deux venant du monde de l'éducation nationale (l'Action Culturelle du Rectorat et l'IUFM - Institut Universitaire de Formation des Maîtres) et deux du monde associatif (le GRAINE, réseau régional d'éducation à l'environnement et l'Ifrée-ORE, structure associative partenariale au sein de laquelle les collectivités jouent un rôle important et elle-même issue d'une histoire partenariale régionale forte).

15 Cette recherche dans le domaine de l'éducation relative à l'environnement (ERE), et en référence à la typologie établie par Lucie Sauvé (1998-1999), se situe plutôt dans le cadre d'une recherche "critique » c'est-à-dire « orientée vers l'action susceptible de produire un savoir critique de nature à catalyser le changement social» et d'une recherche « pour» l'ERE.

16 Étant dans le droit fil de l'histoire de vie d'une équipe de co-formateurs, ses membres se sont portés largement volontaires pour s'impliquer dans le cheminement de cette démarche. Nous avons ainsi été amenés à travailler dans l'esprit d'une recherche-action « centrée sur l'objet partagé » telle que définie par Louis Goffin (1998-1999).

17 L'implication de l'équipe des co-formateurs devenant co-acteurs dans ce projet de recherche m'a certes posé quelques problèmes et m'a obligée à innover et inventer les solutions qui me semblaient les plus adaptées dans ce contexte. Elle a aussi été le cadre des moments les plus forts et riches de ce travail et a instauré une réelle pratique réflexive collective. D'autre part, ce travail avec des regards croisés sur un même sujet de recherche peut aussi être considéré comme favorable à une certaine objectivité par la multiplicité des points de vue considérés.

18 L'accueil des stagiaires pour entrer dans cette recherche-action a été globalement très bon et nous avons pu découvrir de façon encore plus explicite toute la richesse d'un groupe de personnes, venant pour se former, mais aussi tellement détenteur de savoirs à partager. Cette place inhabituelle donnée aux stagiaires a affiché de façon tout à fait claire l'intérêt et le respect que nous portons aux personnes davantage qu'à leurs statuts, elle a introduit cette relation réciproque décrite par Jean-Marie Labelle (1996), cette confiance et cette connivence pour avancer dans une réflexion permanente en vue de l'épanouissement personnel et professionnel de chacun.

\section{Une méthodologie de mise à l'épreuve}

19 Le protocole d'enquête s'appuie sur deux stages nationaux de formation de formateurs, espacés de six mois, et permettant donc au niveau des co-formateurs la promotion d'une réflexion active de façon à mettre à l'épreuve les pratiques développées; il 
considère les différents niveaux de personnes concernées par ce partenariat (responsables institutionnels, formateurs et stagiaires).

Il diversifie les méthodes utilisées :

- enregistrements en situation, relevant donc de l'observation, soit pendant les réunions de travail des formateurs, soit pendant les séquences des stages ;

- entretiens individuels semi-directifs auprès des formateurs et des responsables institutionnels ;

- un entretien collectif réunissant tous les formateurs et deux des responsables institutionnels ;

- questionnaires soumis aux stagiaires en fin de stages et plusieurs mois après ;

- mais aussi, référence aux documents officiels des « contrats de partenariat ».

21 Tout ceci afin de pouvoir croiser lors de l'analyse ce qui relève du « dire » et du « faire ", et de vérifier si les différentes personnes relevant des trois pôles de la figure 2 ci-après ont réellement appris en partenariat.

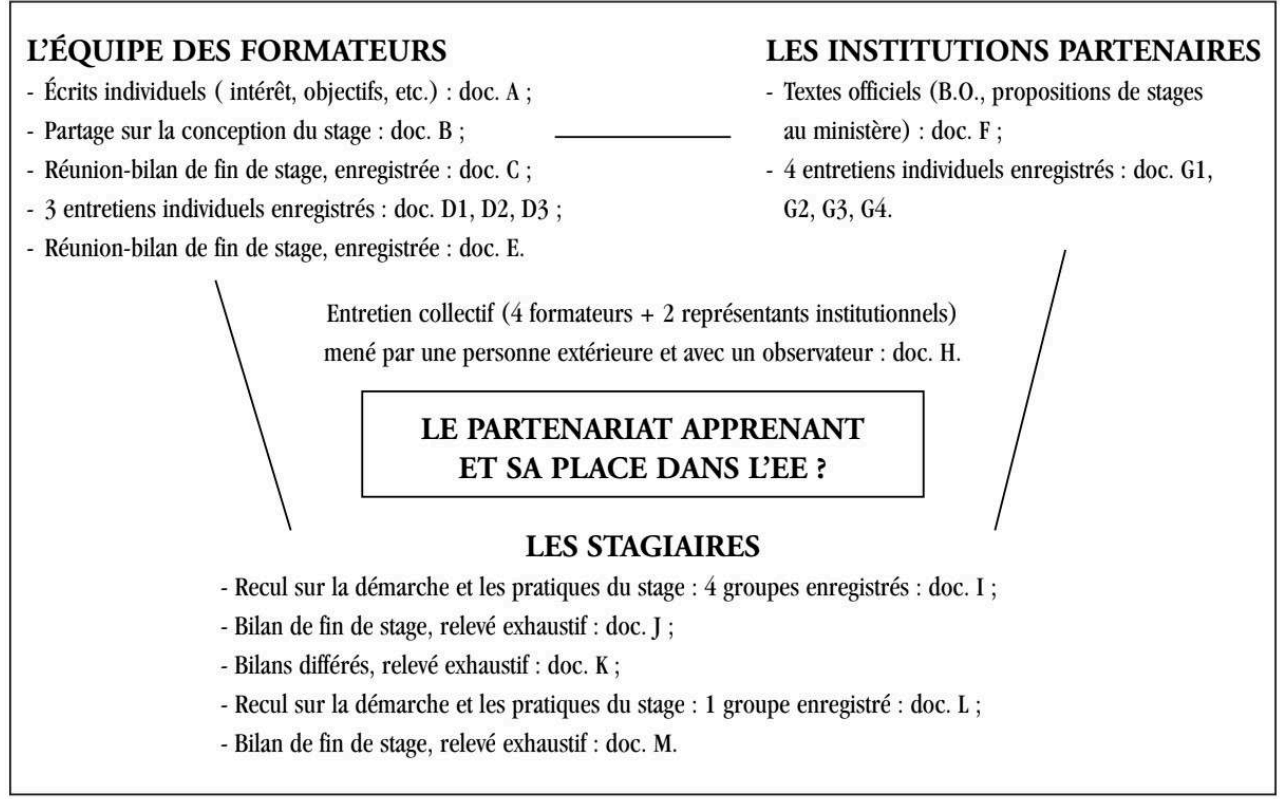

Figure 2 : Récapitulatif des données collectées

\section{Du croisement des données de la recherche à leur interprétation}

\section{L'analyse croisée des résultats}

Afin de mesurer l'apprenante liée au partenariat aux différents niveaux (formateurs, responsables institutionnels, stagiaires, équipe des formateurs, institutions), j'ai réalisé des grilles de lecture croisée des données collectées.

Ainsi, si je prends pour premier exemple le cas de l'équipe des formateurs : il s'est agi de mettre en parallèle les faits constatés, leurs propres dires, le regard des stagiaires et le regard des responsables institutionnels d'abord pendant le premier stage, puis dans la période intermédiaire et enfin lors du deuxième stage. L'apprenante apparaît ainsi très forte au sein de l'équipe des co-formateurs (tableau 1). 
24 Considérons ensuite en second exemple le cas des institutions, dont la faiblesse est déjà repérée au niveau du regard des responsables institutionnels dans le document précédent (tableau 2).

Tableau 1 : Le cas de l'équipe des formateurs

\begin{tabular}{|c|c|c|c|c|}
\hline & D'après les faits & D'après les dires des formateurs & D'après le regard des stagiaires & $\begin{array}{l}\text { D'après le regard des } \\
\text { responsables institutionncls }\end{array}$ \\
\hline $\begin{array}{l}\text { Premier } \\
\text { stage }\end{array}$ & 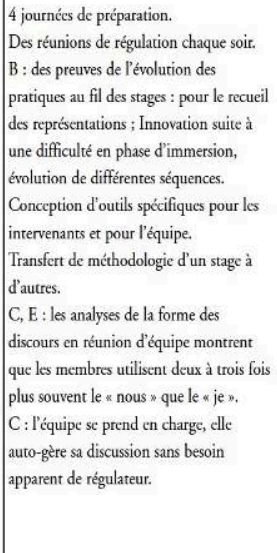 & 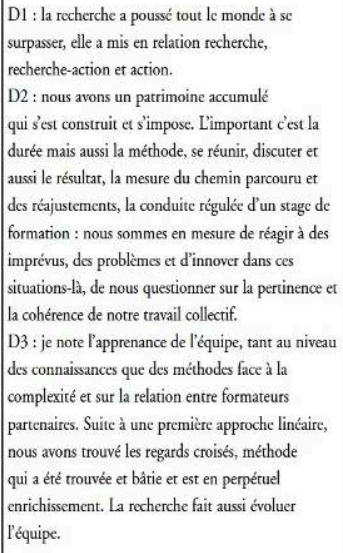 & 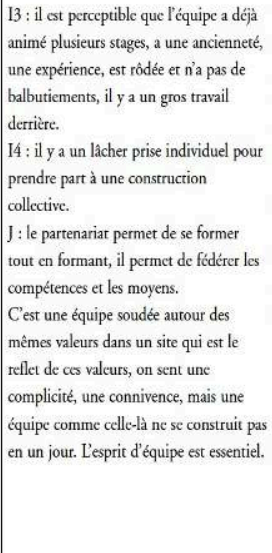 & $\begin{array}{l}\text { G1 : l'́ćquipe construit tet navigue } \\
\text { dans le cadre fixé instiututionnelle- } \\
\text { ment. } \\
\text { G2 : l'́́quipe a la capacité de faire } \\
\text { mieux que la simple juxtaposition ou } \\
\text { addition des compétences, il y a une } \\
\text { valeur ajoutée importante. } \\
\text { G4 : les aspects organisationncls ne } \\
\text { sont pas du tout accessoires, organiser } \\
\text { la vic du partenariat, les réunions, le } \\
\text { calendrier, la conduite des réunions, le } \\
\text { rôle de chacun. }\end{array}$ \\
\hline
\end{tabular}

Tableau 1 : Le cas de l'équipe des formateurs (suite)

\begin{tabular}{|c|c|c|c|c|}
\hline & D'après les faits & D'après les dires des formateurs & D'après le regard des stagiaires & $\begin{array}{l}\text { D'après le regand des } \\
\text { responsables institutionnels }\end{array}$ \\
\hline \begin{tabular}{|l|} 
Perriode \\
intermédiaire
\end{tabular} & $\begin{array}{l}\text { C: recherche de remédiation pour une } \\
\text { meilleure ges tion du temps, ou pour la } \\
\text { recherche de plus d'alternance. } \\
\text { Des modifications ou des pistes } \\
\text { complètement nouvelles. }\end{array}$ & $\begin{array}{l}\text { C: notre équipe n’est pas un bloc, c'est un } \\
\text { assemblage. Elle a une meilleure maâtrise des } \\
\text { problèmes, est plus sereine plus apte à gérer les } \\
\text { conflits et les frustrations. }\end{array}$ & & \\
\hline $\begin{array}{l}\text { Detasième } \\
\text { stage }\end{array}$ & $\begin{array}{l}4 \text { journées de prépararion et perites } \\
\text { réunions par groupes. } \\
\text { Pas de réunions spéáfiques de } \\
\text { concertation pendant le stage } \\
\text { (juste des petis moments rapides). } \\
\text { Moins de fatigue et de stress. }\end{array}$ & 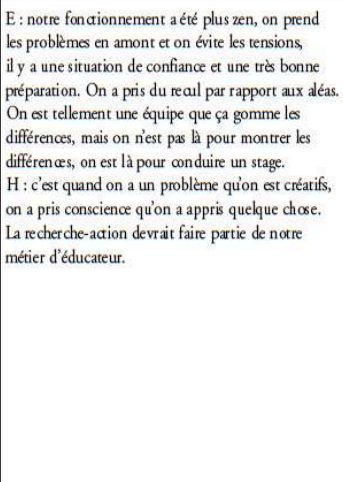 & 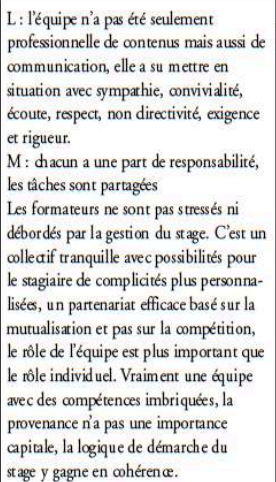 & $\begin{array}{l}\text { Auaun retour des institutionnels après } \\
\text { l'envoi des evaluations }\end{array}$ \\
\hline
\end{tabular}


Tableau $2:$ Le cas des institutions

\begin{tabular}{|c|c|c|c|c|}
\hline & D'après les faits & $\begin{array}{c}\text { D'après les dires } \\
\text { des responsables institutionnels }\end{array}$ & $\begin{array}{l}\text { D'après les regards } \\
\text { des formateurs }\end{array}$ & D'après le regard des stagiaires \\
\hline Institution 1 & $\begin{array}{l}\text { Peu de communication et de retombés } \\
\text { au sein de línstitution dans son ensemble. }\end{array}$ & $\begin{array}{l}\text { H : cette institution est militante par définition ; Il } \\
\text { émet des doutes sur les possibilitiés d'apprenance de } \\
\text { l'éducarion nationale, la place et le poids de la } \\
\text { personne dans son institution sont des élements } \\
\text { décisifs et la reconnaissance de l'importance du } \\
\text { partenariat par l'institution est déćisive. } \\
\text { E : c'est aux personnes de venir śinformer sur ce } \\
\text { qu'est notre institution, nous sommes disponibles. }\end{array}$ & $\begin{array}{l}\text { D1 : des pratiques nouvelles d'écriture et } \\
\text { de présentation aux stagiaires de la } \\
\text { conception et de la préparation des } \\
\text { stages ont été transfétés. } \\
\text { Mais pour le réseau dans son ensemble, } \\
\text { il n'y a pas vraiment de rénvestissement. } \\
\text { H: il y a plus de travail pour que } \\
\text { l'éducation nationale soit apprenante. }\end{array}$ & 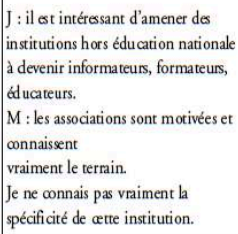 \\
\hline Institution 2 & $\begin{array}{l}\text { Une démarche concertée mais provoquéé } \\
\text { par linss sitution } 2 \text { auprès des institutions } \\
3 \text { et } 4 \text { afin de déboucher sur une } \\
\text { convention de partenariat et un } \\
\text { programme concret d'actions (impliquant } \\
\text { aussi linstitution 1). }\end{array}$ & $\begin{array}{l}\text { G2 : il s'agit d' une expérimentation intéressante, } \\
\text { mais I2 se pose de questions de gestionnaire } \\
\text { (côut en énergie, en temps, en moyens). }\end{array}$ & $\begin{array}{l}\text { E: les personnes qui souhaitaient nous } \\
\text { connaitre davantage sont venues le } \\
\text { demander. } \\
\text { H: il faudrait être plus attentif aux } \\
\text { formu lations des partenariats. } \\
\text { Il est important de travailler sur le } \\
\text { partimoine de ran coeur d'image, de } \\
\text { représentarions de chaque institution. } \\
\text { Mon instirution a bien pris en com pte le } \\
\text { parten ariar, c'est une institution jeune } \\
\text { qui évolue, le partenariat idéal c'est celui } \\
\text { qui fait evoluer les partenaires. }\end{array}$ & $\begin{array}{l}\text { M: je ne connais pas vraiment la } \\
\text { spócificité de cette institution. }\end{array}$ \\
\hline
\end{tabular}

Tableau 2 : Le cas des institutions (suite)

\begin{tabular}{|c|c|c|c|c|}
\hline & D'après les faits & $\begin{array}{l}\text { D'après les dires } \\
\text { des responsables institutionnels }\end{array}$ & $\begin{array}{l}\text { D'aprè̀ les regards } \\
\text { des formateurs }\end{array}$ & D'après le regard des stagiaires \\
\hline Institution 3 & $\begin{array}{l}\text { Acceptation du dialogue suite aux stages, } \\
\text { bon investisement au niveau de la } \\
\text { direction de linstitution, mise en forme } \\
\text { d'un programme. }\end{array}$ & $\begin{array}{l}\text { G3 : affirmation de la volonté politique de } \\
\text { linstiturion de s'engager (financièrement et } \\
\text { implication personnelle) dans le partenariat. } \\
\text { I3 souhaiterait voir s'installer une dynamique dans } \\
\text { son institution, il pense que ces expériences res tent } \\
\text { ignorés. }\end{array}$ & $\begin{array}{l}\text { H: l'institution apprend, même si elle le } \\
\text { fait avec ses pesanteurs dues à l'́ćchelle. } \\
\text { Quand on voit quiune stagiaire a mis en } \\
\text { place un stage de ce type dans son propre } \\
\text { IUFM, avec des gens avec lesquels elle ne } \\
\text { travaillait pas, oui l'institution apprend. }\end{array}$ & 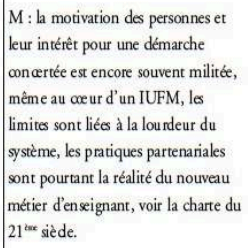 \\
\hline Institution 4 & $\begin{array}{l}\text { Grấce à la démarche concertée avec } \\
\text { l'institution } 2 \text {, une meilleure } \\
\text { relation entre les institutions } 3 \text { et } 4 \text {. }\end{array}$ & $\begin{array}{l}\text { G4 : I I voit des retombés en gain de } \\
\text { compétences et d'efficacité pour toute } \\
\text { Pinstitution, élèves, enseignants établissements, une } \\
\text { reconn aissance instiutionnelle de notre Académie et } \\
\text { une mod fifcation du regard des autres partenaires sur } \\
\text { PEN. Il pense qu'on dev rait organiser le rayonnement } \\
\text { de ces svoir faire de façon plus systématique et en } \\
\text { interne. }\end{array}$ & $\begin{array}{l}\text { H : Les changements doivent venir de la } \\
\text { base en interne ; importance de mener } \\
\text { des expérience à Pintérieur du système } \\
\text { mais aussi de faire des alliances avec } \\
\text { Pextérieur : intérêt stratégique et militant } \\
\text { du partenariat. } \\
\text { Quelques individus peuvent cróer une } \\
\text { étincelle décisive. }\end{array}$ & $\begin{array}{l}\mathrm{J} \text { : le partenariat est un espoir } \\
\text { dévolution du système soolaire, } \\
\text { c'est la solution pour vaiment } \\
\text { dédoisonner les disciplines. } \\
\text { Il faut attendre moins des } \\
\text { institutions et renforcer son } \\
\text { engagement personnel, nous devons } \\
\text { obliger les instiututions à darifier leur } \\
\text { position, notamment l'EN. }\end{array}$ \\
\hline
\end{tabular}

\section{Une interprétation par la confrontation aux travaux de Nonaka et Takeuchi (1997)}

Cette confrontation a cherché à appréhender le système partenarial dans sa globalité donc à considérer d'une part la démarche mise en œuvre par les formateurs euxmêmes au sein de leur équipe, d'autre part la démarche proposée aux stagiaires mais aussi à prendre en compte le niveau des responsables institutionnels.

La recherche de la spirale de création de connaissances au niveau de l'équipe des formateurs (figure 3 et se reporter à la figure 1) 


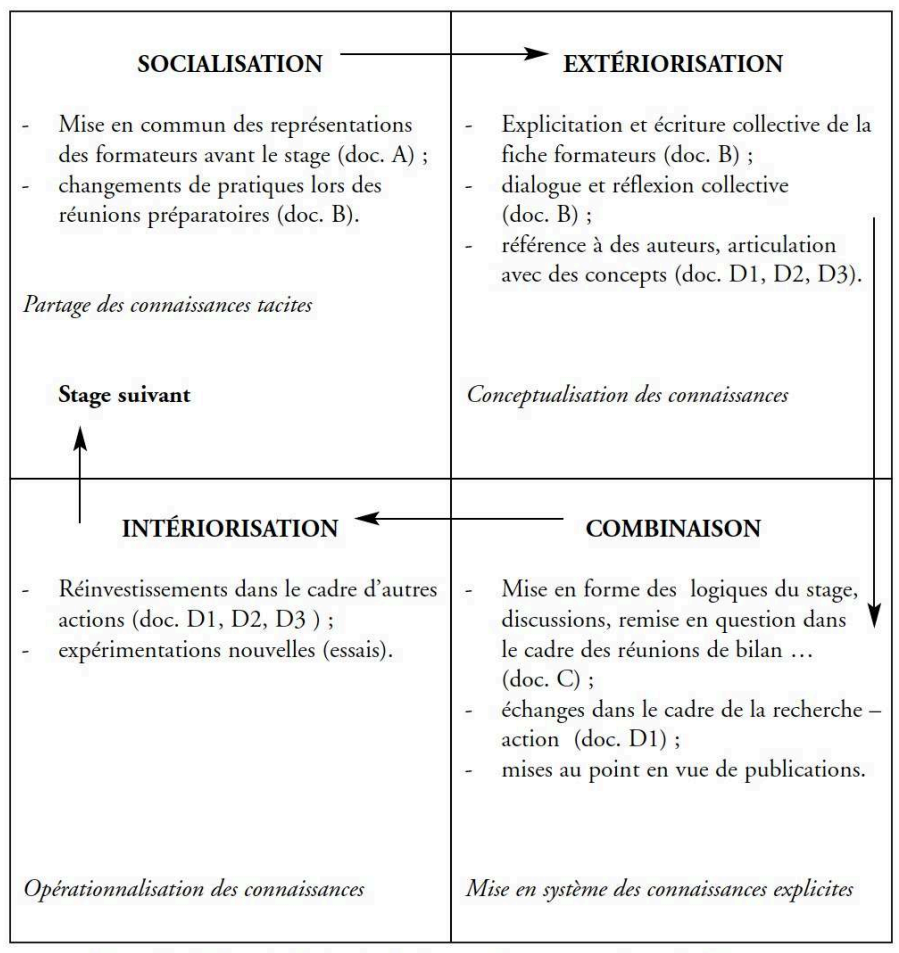

Figure 3 : Recherche de la spirale de connaissances au niveau des formateurs

Si nous cherchons à caractériser la place du partenariat dans cette spirale, nous pouvons dire que :

- la phase de socialisation se trouve enrichie par la diversité des talents acquis par les formateurs dans des contextes institutionnels différents. La confrontation des représentations et le partage des connaissances tacites couvre donc, grâce au partenariat, un champ beaucoup plus large.

- lors de la phase d'extériorisation, les apports conceptuels se trouvent multipliés par la diversité des sources et des apports liés à chaque formateur.

- en cas de crise (de " chaos » selon Nonaka et Takeuchi, 1997), la gestion collective du problème est selon les dires des formateurs, plus confortable, plus efficace et renforce les liens du groupe.

- un frein éventuel se situe au niveau du temps nécessaire pour mettre en œuvre toutes ces étapes (parfois, on irait plus vite tout seul, dit un formateur, mais seulement si on considère le court terme ajoute-t-il).

Ainsi, dans le cadre de la conception, de la préparation et du déroulement des stages, l'équipe des formateurs se donne les moyens de vivre au travers de ses échanges les étapes de cette spirale et crée des connaissances nouvelles qui, une fois intériorisées, seront réinvesties dans le stage suivant ou dans d'autres actions. Il s'agit donc bien d'une équipe apprenante qui en quelque sorte a " inventé » cette spirale sans en avoir la connaissance théorique. La révélation des écrits de Nonaka et Takeuchi (1997) devient pour elle un support de réflexion supplémentaire. À noter qu'au travers de cette démarche, les formateurs ont souhaité s'appliquer à eux-mêmes la démarche qu'ils mettent en œuvre avec les stagiaires, comme par exemple s'imposer, malgré leur habitude de travailler ensemble, une phase de recueil de leurs représentations au début de chaque nouvelle action. 
29 L'importance et la place de cette recherche-action ont été abondamment signalées par les personnes partenaires ce qui a aidé à visualiser et formuler l'apprenante au cœur de ce partenariat.

\section{La recherche de la spirale de création de connaissances au niveau des stagiaires} (figure 4)

Au niveau des stagiaires, l'influence du partenariat peut ainsi être repérée :

- les rencontres organisées dans le cadre de la phase d'immersion ou de celle des regards simultanés sont enrichies par la multiplicité des partenaires et par leur vigilance (pour couvrir par exemple tous les « pouvoirs » représentés)

- le choix des formateurs de considérer que les stagiaires sont partenaires de ce système mis en place pour la formation de formateurs et de les associer à la recherche-action développe les dialogues coopératifs (phase de recul, moment de partage des outils mis en place pour l'organisation du stage, etc.) ;

- le fait de faire vivre ce partenariat au lieu de simplement dire qu'il est bien de travailler en partenariat semble essentiel (mélanger les stagiaires formateurs de l'éducation nationale et hors éducation nationale, prouver que les formateurs sont réellement partenaires et que c'est efficace, etc.) ;

- le frein évoqué par les stagiaires est souvent d'être capables de " trouver » les personnes avec qui faire équipe, de bénéficier d'un soutien de leur institution et aussi de trouver le temps pour s'y consacrer.

31 Ainsi, il apparaît possible de dire que les stagiaires, dans le cadre de leur semaine de formation par la démarche qui leur est proposée, sont en mesure de vivre les étapes de la spirale de création de connaissances, sachant que la dernière phase d'intériorisation se poursuivra bien au-delà du stage. Ils disent eux-mêmes dans les phases de recul et dans leurs évaluations être étonnés de la richesse produite par le groupe ; il s'agit donc bien au delà des connaissances apportées et prévues, de création de connaissances nouvelles. 


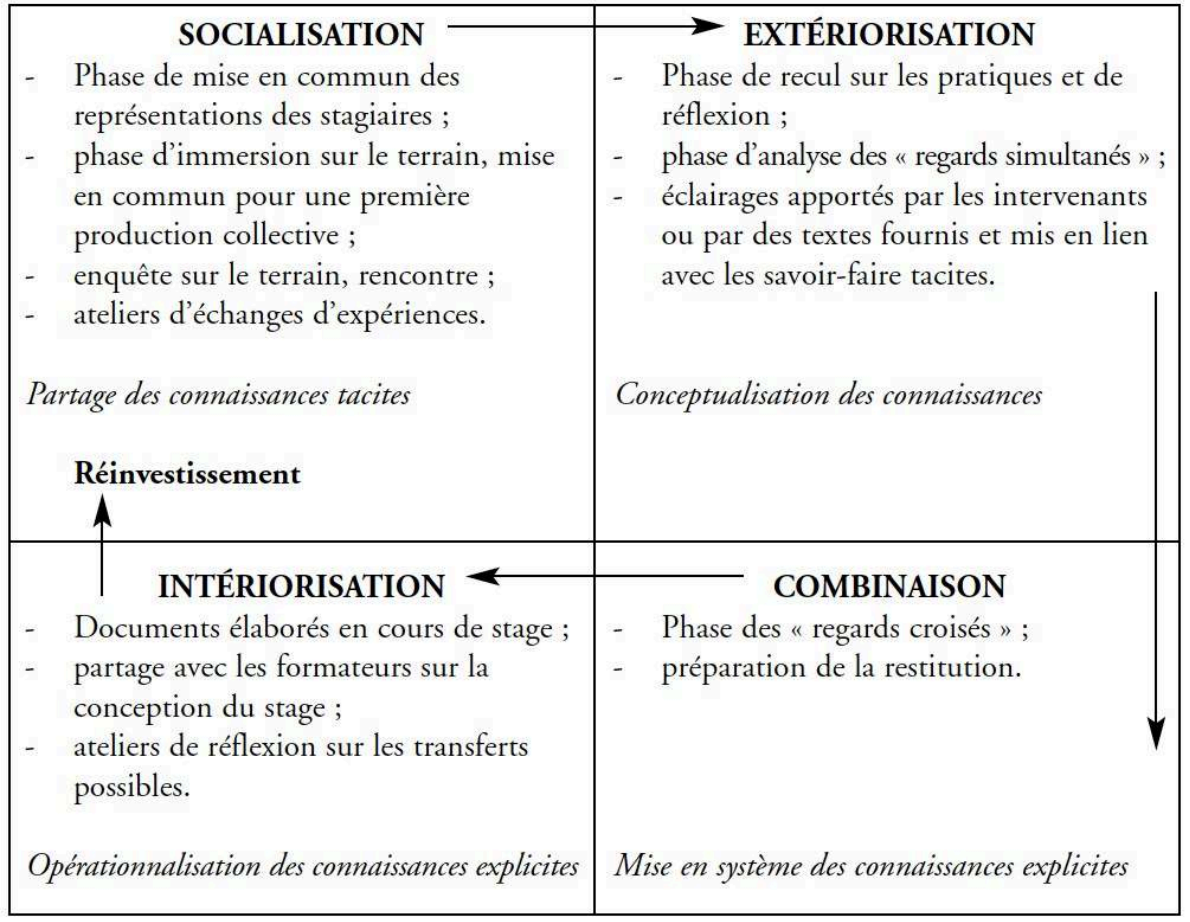

Figure 4 : Recherche de la spirale de connaissances au niveau des stagiaires

Cette spirale vécue par les stagiaires, bien que fondée sur le même principe que celle vécue par les formateurs, n'est pas confondue avec celle-ci ; j'y vois davantage une double spirale vécue par chaque groupe de personnes en fonction de sa position mais avec des possibilités d'échanges notamment lors de phases de recul et de réflexion.

\section{Au niveau des responsables institutionnels}

Force est de constater que, dans le cas présent, les responsables institutionnels constituent un groupe virtuel dans la mesure où ils sont co-responsables de cette action de formation mais ne se rencontrent pas. La seule occasion de rencontre, provoquée dans le cadre de cette recherche (donc de nature exceptionnelle), a été l'entretien collectif (doc. H) auquel deux des quatre représentants institutionnels ne sont pas venus.

4 En fait, on constate qu'ils s'appuient d'une part sur le document officiel (conçu en grande partie par les formateurs) qu'ils ont validé individuellement (doc. F) et d'autre part sur leurs relais que sont les formateurs de chaque institution à qui ils délèguent leur pouvoir (ce qui contribue au mélange des rôles). Ils reçoivent des informations sur l'avancement du projet (planning des stages, etc.), mais ne croisent jamais ces informations entre eux. C'est donc un partenariat plus passif que celui de l'équipe des formateurs, un partenariat qui existe sur le papier, un " partenariat décrété » (et c'est important, au moins symboliquement comme preuve de reconnaissance, comme le fait remarquer un des responsable associatif), mais qui ne correspond pas à une action commune entre les responsables institutionnels.

Il n'est donc pas possible dans ce cas de chercher à construire une spirale de création de connaissances avec les données dont nous disposons.

Ceci ne veut toutefois pas dire que les responsables institutionnels n'évoluent pas dans leurs propres conceptions autour de ces stages car en fonction des relations qu'ils ont 
avec leur formateur et du degré d'écoute et de confiance qu'ils ont envers lui, ils peuvent aussi " avancer » (doc. G4), mais ils ne sont pas dans cette situation euxmêmes producteurs de connaissances. On peut toutefois se demander si cela correspond à un réel choix de leur part et s'ils ont vraiment eu la possibilité d'entrer dans cette démarche de partenariat actif.

\section{La prise en compte du système partenarial global}

Cette étude nous montre combien il est important de considérer le "système partenarial " dans son ensemble, d'en déterminer tous les acteurs sans oublier les institutions dont chacun dépend et qui ont forcément une influence mais aussi les bénéficiaires du partenariat, ceux pour qui il a été mis en place, et les relations qui existent entre ces éléments du système.

La construction d'un schéma représentant ce système peut, tout comme l'écriture, " aider à penser » et permettre de voir, comme dans le cas décrit ci-dessus, que certains sont plus " acteurs » et d'autres plus "figurants ». On pourra ainsi distinguer un partenariat " actif » et un partenariat " passif », et chercher à œuvrer pour rendre le système global plus actif.

En référence aux travaux de Daniel Sibony (1991) sur la notion d'" entre-deux ", nous avons pu montrer que chaque personne dans ce jeu partenarial a sa façon de gérer les entre-deux, d'en faire de simples liaisons, des interactions ou des transactions. C'est une affaire d'engagement, d'approches, de frontières que l'on ouvre ou referme, de relations dans lesquelles on accepte ou pas d'entrer (on voit là que l'expression " entrer en partenariat " prend tout son sens).

Ainsi pouvons-nous dire que, si le partenariat établit bien comme principe de base la mise en place d'interrelations, il ne garantit pas à coup sûr la mise en place d'interactions authentiques; on peut très bien " être " en interrelation sans pour autant « faire » en interrelation, c'est-à-dire sans interagir, sans qu'il se passe quelque chose dans la zone d'entre-deux.

41 Nous rejoignons là l'idée qu'une politique partenariale peut tout à fait se décréter, mais que par contre, les pratiques partenariales ne peuvent se décréter et doivent s'instaurer ensemble grâce à l'énergie produite par la relation entre les personnes.

Ainsi, le partenariat apprenant existe, il permet par la combinaison des compétences d'obtenir des résultats inaccessibles autrement. Il est résolument constructiviste et laisse une grande place aux personnes, à leur envie d'apprendre ensemble, de partager la réflexion dans et sur l'action, à être comme le dit Donald Schön (1994) des " praticiens réflexifs ». Mais, le partenariat apprenant, nous l'avons vu, nécessite de l'espace et du temps, il ne peut être " pressé »! Il ne doit toutefois pas être considéré comme une panacée car dans certains cas, si le contexte est vraiment mauvais ou si les personnes ne sont pas motivées, il peut être seulement grand consommateur de temps sans grands bénéfices. En effet, bien que le partenariat impose des interrelations, il ne garantit pas à coup sûr la mise en place d'interactions authentiquement apprenantes ! 


\section{Vers un partenariat apprenant de qualité pour plus de cohérence}

\section{À la recherche des indicateurs de qualité du partenariat apprenant}

43 doivent être pris en considération dans le cadre d'actions partenariales:

- le contexte de chaque institution partenaire (une association étant ici référée comme institution) mais aussi celui de l'équipe impliquée professionnellement dans l'action et de chaque personne en particulier, déterminant "un » contexte global qui dépend bien évidemment fortement des actions passées, de leurs singularités, des histoires individuelles... et peut être favorable ou non au regard du projet.

- les interventions des personnes sur lesquelles le partenariat repose et prend appui, leur place, leur posture et leurs interventions aussi bien en tant que responsables institutionnels, en tant que membres d'une équipe ou à titre individuel.

- les processus mis en place relativement au projet et relativement au partenariat, encore une fois aussi bien par les institutions, par l'équipe qui a en charge leur mise en œuvre que par les personnes elles-mêmes.

- les résultats obtenus relativement au projet mais aussi relativement à la qualité du partenariat mis en œuvre, au niveau des institutions, au niveau de l'équipe et au niveau de chaque personne.

Il s'agit là d'un faisceau de conditions qui interagissent et qui en fonction de leur pertinence vont déterminer le fait que le partenariat " marche » (qu'il soit efficace et efficient) et éventuellement, si le nouveau contexte est favorable, rebondisse et « dure $\gg$.

Certains points ont ainsi pu être dégagés comme essentiels pour une réussite du partenariat et nous ont amenés assez souvent à mettre en forme des stratégies ou des outils spécifiques :

- l'expression des attentes de chacun et de ses intérêts dans le partenariat est pour nous un moment essentiel qui nécessite qu'on lui consacre du temps. Ainsi au début de chaque projet, et bien que très souvent nous nous connaissions déjà, nous consacrons un temps à l'expression de nos représentations face à ce nouveau projet, à l'idée que nous nous faisons de notre place et de celle des autres au sein du projet partenarial ; temps d'abord d'écriture individuelle puis de mise en partage dans un tour de table. Cette pratique nous permet d'éviter bien des malentendus, de prendre en considération l'ensemble des attentes mais aussi parfois d'exprimer un certain patrimoine de rancœurs pouvant faire obstacle (et l'éducation nationale tout comme les associations sont de bonnes cibles de toutes ces représentations très connotées qui nous viennent de loin !).

- l'affichage officiel du partenariat, et de tous les partenaires à égalité, ne nous semble vraiment pas être un point de détail mais bien un indicateur d'une forme du respect et de l'estime mutuelle que se doivent les partenaires quel que soit leur poids institutionnel en dehors de ce partenariat. Aucune prépondérance révélant une forme de domination ne nous semble acceptable (taille des logos, formulations utilisées par exemple. On trouve encore trop souvent des expressions telles que « en partenariat avec..., X organise... » au lieu de mentionner une réelle co-organisation). Ces points sont à aborder dès la mise en place du partenariat et peuvent éventuellement amener à une rupture de contrat si les évolutions sont impossibles, ou alors à une clarification précisant qu'il ne s'agit plus d'un partenariat 
mais clairement d'une sous-traitance ou d'une simple intervention (ce qui n'est pas une honte loin de là, si les choses sont claires). Ils sont à mettre en relation avec la notion de responsabilité partagée qui passe par le partage des risques et des bénéfices l'accueil et la gestion collective des problèmes.

- une bonne mise en circulation des informations révélatrice d'une volonté de partage du pouvoir, ainsi qu'une répartition claire des rôles et des tâches de chacun nous semblent devoir passer obligatoirement par des écrits (dans notre culture occidentale en tout cas) validés par tous et circulant le plus rapidement possible (et là, l'outil du courrier électronique permettant l'envoi de fichiers s'est avéré être un apport important). Ainsi, par exemple, dans la préparation de nos formations, un document papier est mis en forme collectivement comme outil de travail mais aussi document de référence, avec pour chaque séquence du stage une phrase résumant les objectifs pédagogiques ainsi que les moyens à mobiliser et mettre en œuvre pour y répondre et enfin qui fait quoi.

- la mise en place de "temps de prise de recul ", de regard sur le vécu se rapprochant des techniques d'analyses de pratiques, nous semble essentielle si on veut entrer dans la dynamique et le plaisir du « partenariat apprenant » créant des connaissances nouvelles, accueillant les critiques et les problèmes, mais aussi initiant de la connivence. Notre expérience a montré la mise en place de ces temps de recul au niveau de l'équipe des formateurs pendant et après les formations mais aussi avec les stagiaires ; par contre ils n'ont pas été organisés avec les représentants institutionnels et on peut constater qu'ils ont cruellement manqué.

- le rayonnement par la mise en relation de l'expérience partenariale avec le travail au sein de chaque institution relève de l'implication des personnes (responsables institutionnels et membres de l'équipe) mais aussi des possibilités laissées par l'institution ; il a permis dans certains cas la valorisation des résultats obtenus et des démarches mises en œuvre pour y parvenir, entraînant des retombées en interne pour chacun et de nouvelles compétences. Il est, nous semble-t-il, déterminant pour l'avenir du partenariat.

C'est donc bien de la qualité du partenariat mis en place et de son évaluation collective que dépendent sa reconduction et sa consolidation, ou au contraire sa dislocation.

Ainsi sommes-nous convaincus que ces nouvelles pratiques, compte tenu de notre culture, sont loin de nous être naturelles ! Elles s'apprennent et se construisent au fil des expériences, à condition qu'on accepte de leur consacrer du temps, le temps nécessaire à leur maturation, le temps que cette spirale s'élève ! Cela implique que chacun soit intéressé à entendre le point de vue de l'autre, à prendre en considération toutes les compétences, qu'il accepte de partager le contrôle de l'action et de prendre le risque d'expérimenter et de créer des occasions, de réfléchir pendant l'action pour que cesse enfin le jeu du « si je gagne, tu perds ».

\section{La cohérence des pratiques du partenariat apprenant en éducation à l'environnement}

Lucie Sauvé (2000) dégage trois catégories d'arguments en faveur du partenariat en ERE: un argument épistémologique, c'est-à-dire relevant du processus même de production des savoirs, un argument éthique lié à la discussion démocratique autour de l'environnement en tant qu'objet politique (qui concerne les choses publiques), et enfin un argument stratégique de mise en convergence des ressources pour un projet commun allant vers un changement environnemental, social et éducationnel. Pour ma 
part, j'ai tenté, dans le tableau 3 qui suit, d'établir un parallèle entre les pratiques partenariales et les objectifs de l'éducation à l'environnement.

Tableau 3 : La mise en relation des pratiques partenariales et des objectifs de l'EE

\begin{tabular}{|c|c|c|}
\hline $\begin{array}{l}\text { Les pratiques du } \\
\text { partenariat apprenant }\end{array}$ & Intérêts éducatifs & $\begin{array}{l}\text { Intérêts pour l'éducation à } \\
\text { l'environnement }\end{array}$ \\
\hline $\begin{array}{l}\text { Mettre en commun ses envies, ses } \\
\text { questions, ses objectifs. }\end{array}$ & $\begin{array}{l}\text { Constituer une communauté } \\
\text { d'apprentissage. }\end{array}$ & $\begin{array}{l}\text { Percevoir une communauté de } \\
\text { préoccupations autour de l'ERE. }\end{array}$ \\
\hline $\begin{array}{l}\text { Exprimer ses représentations du projet } \\
\text { et de ses compétences par rapport aux } \\
\text { autres. }\end{array}$ & $\begin{array}{l}\text { Se situer en tant que personne et } \\
\text { professionnel : renforcer son identité et } \\
\text { développer son autonomie. }\end{array}$ & $\begin{array}{l}\text { Se percevoir et se situer comme l'un } \\
\text { des acteurs d'un tout. Être au clair } \\
\text { avec soi-même avant d'être bien avec } \\
\text { les autres et avec le monde. }\end{array}$ \\
\hline $\begin{array}{l}\text { Connaître le contexte global du projet } \\
\text { partenarial. }\end{array}$ & $\begin{array}{l}\text { Première phase d'une approche } \\
\text { systémique : "s'élever pour mieux voir ". }\end{array}$ & $\begin{array}{l}\text { Savoir prendre en compte tous les } \\
\text { éléments d'un monde « infiniment } \\
\text { complexe ". } \\
\text { Insister sur le caractère } \\
\text { interdisciplinaire de l'ERE. }\end{array}$ \\
\hline $\begin{array}{l}\text { Pratiquer les " regards croisés ". } \\
\text { Accepter différentes façons de faire, } \\
\text { différents rythmes, différents modes } \\
\text { de pensée. }\end{array}$ & $\begin{array}{l}\text { Accepter les différences. Mettre à profit } \\
\text { la diversité. Partager les savoirs, les faire } \\
\text { dialoguer, "relier pour mieux } \\
\text { comprendre". }\end{array}$ & $\begin{array}{l}\text { Approcher l'« infiniment } \\
\text { préoccupant " sur le principe d'une } \\
\text { discussion démocratique. }\end{array}$ \\
\hline $\begin{array}{l}\text { Arriver à une production commune } \\
\text { négociée. }\end{array}$ & $\begin{array}{l}\text { Développer des savoir-agir ensemble } \\
\text { "s'impliquer plutôt qu'être impliqué ". }\end{array}$ & $\begin{array}{l}\text { Prendre en compte l'environnement } \\
\text { en tant qu'objet « socialement } \\
\text { construit » et évolutif. }\end{array}$ \\
\hline $\begin{array}{l}\text { Répondre collectivement à l'imprévu } \\
\text { des situations. } \\
\text { Innover. }\end{array}$ & $\begin{array}{l}\text { Développer l'habileté de fonctionner } \\
\text { ensemble, la créativité et une certaine } \\
\text { sérénité face aux difficultés. }\end{array}$ & $\begin{array}{l}\text { Renforcer l'idée qu'il n'y a jamais de } \\
\text { solutions toutes prêtes. Développer la } \\
\text { prise de responsabilités partagées. }\end{array}$ \\
\hline $\begin{array}{l}\text { Développer des temps de prise de } \\
\text { recul, s' interroger sur ses } \\
\text { pratiques. }\end{array}$ & $\begin{array}{l}\text { Devenir des " praticiens réflexifs " par } \\
\text { le dialogue. }\end{array}$ & $\begin{array}{l}\text { S'interroger sur ses actions, développer } \\
\text { une vision sur leurs conséquences à } \\
\text { long terme. }\end{array}$ \\
\hline Évaluer collectivement. & $\begin{array}{l}\text { Développer le sens de l'évaluation } \\
\text { prospective. }\end{array}$ & Assumer une responsabilité partagée. \\
\hline
\end{tabular}
compte le souci de l'apprenante des partenaires, s'inscrivent totalement dans les objectifs éducatifs contemporains et qu'elles favorisent l'intégration du savoir-agir caractéristique de l'éducation à l'environnement pour que les savoirs deviennent de réelles compétences dans ce domaine.

ariat est vraiment au cœur du système éducatif ", il est pertinent, utile et nécessaire. Je suis en accord avec ce point de vue, j'ajouterais juste une réserve : à condition qu'il s'agisse d'un véritable partenariat apprenant et non de situations partenariales plaquées comme on a pu en voir trop souvent dans notre système éducatif et qui ont desservi l'idée que le partenariat pouvait avoir une fonction éducative.

\section{Quels sont les atouts et les limites de cette notion de partenariat apprenant ?}

\section{Le partenariat apprenant est lié aux personnes}

Il est lié à la conviction que chacun a de l'intérêt à construire des partenariats ce qui comme le dit l'un des formateurs au départ plutôt enclin à un travail solitaire (doc. D1), peut demander des efforts personnels pour parvenir vraiment à une construction commune.

52 Il est lié aux plaisirs d'un travail en équipe soulignés par tous les formateurs de l'équipe, à l'émergence de richesses nouvelles étonnantes qui permettent à chacun de progresser (doc. A). 


\section{personnes ce qui constitue à la fois un
maintenant le niveau des institutions.} amener une institutionnalisation des pratiques partenariales. Ainsi, comme le dit la formatrice de l'IUFM (doc. H): "c'est bien cette institution qui nous permet d'apprendre ensemble et de faire bouger les choses, c'est en train de se faire et l'institution apprend aussi même si elle le fait avec ses pesanteurs " et elle étaye sa démonstration en disant que d'autres collègues ont été entraînés par son témoignage et ont proposé à leur tour des stages en partenariat dans le plan de formation. On a donc bien dans ce cas une institution qui entre dans une dynamique qui ne lui était pas a priori habituelle. Les autres institutions impliquées dans notre expérience sont politiquement définies autour de l'idée de partenariat : ce sont les textes fondateurs de l'action culturelle dans l'Éducation nationale, ce sont également ceux de l'Ifrée-ORE, structure à vocation partenariale, et ceux construits par le GRAINE dans le cadre de sa charte de qualité de l'éducation à l'environnement.

Une fragilité toutefois car dans des systèmes hiérarchiques comme ceux de l'Éducation nationale des pratiques instituées au fil des années peuvent être remises en cause rapidement par la volonté d'un supérieur hiérarchique ou par des contraintes administratives. Mais fragilité aussi pour les autres cas, y compris pour des structures en réseau, car le partenariat apprenant nécessite que l'on s'occupe de lui, qu'on l'entretienne. Ainsi les contraintes économiques d'une association qui empêcheraient une implication totale dans la démarche, ou encore une non acceptation par les personnes de remettre en question leurs certitudes et leurs savoir-faire établis, 
mèneraient à réduire l'apprenante comme cela a été pour le GRAINE dans notre recherche-action.

61 Une institution est apprenante lorsque les personnes qui la constituent ont compris qu'elles doivent instituer un système de partage et de diffusion, c'est semble-t-il le cas pour l'Ifrée-ORE dont le formateur dit que c'est une jeune institution qui prend bien en compte le partenariat et qui a évolué (doc. $\mathrm{H}$ ).

C'est bien au niveau de la mise en place de ces procédures de mutualisation des connaissances nouvelles que semble se situer le problème essentiel pour les institutions. Ainsi, comme le dit Pierre Calame (1996) "Les institutions sont généralement amnésiques. Elles savent mal et sont parfois même peu soucieuses de gérer leur mémoire, de la socialiser, de construire une connaissance collective à partir de l'expérience » et Donald Schön (1994, p. 383-397) nous fournit également une piste de réflexion dans ce sens lorsqu'il distingue deux types d'institutions avec, je trouve, deux belles formules, les qualifiant d'« institutions sympathiques à la pratique réflexive » ou d'« institutions dynamiquement conservatrices ».

Des pistes de travail complémentaire à cette première approche se dessinent, et pourront peut-être être développées dans le cadre de recherches futures, notamment :

- quelles articulations possibles entre des logiques de réseaux et des logiques hiérarchiques?

- quels mythes du partenariat dans nos sociétés contemporaines?

- une mise en perspective avec d'autres apports théoriques sur les apprentissages organisationnels.

\section{Une antinomie apparente dans la formulation}

64 On pourra trouver une antinomie dans la formulation du " partenariat apprenant " notamment au niveau temporel, l'apprenante impliquant la durée alors qu'un projet partenarial est par définition limité dans le temps.

En effet, les auteurs qui m'ont amenée à cette hypothèse du partenariat apprenant utilisent eux cette formule pour des structures présentant une certaine stabilité dans le temps comme c'est le cas par exemple pour une entreprise.

Est-il donc possible de soutenir cette idée de partenariat apprenant ? Selon moi, l'antinomie est seulement apparente, car si les projets sont bien évidemment limités dans le temps et doivent l'être, l'esprit partenarial instaurant des relations nouvelles de collaboration et un jeu nouveau de partage peuvent par contre être durables de même que les types de processus mis en place.

C'est en développant des procédures communes, en répartissant les responsabilités que le partenariat peut être assimilé à une forme d'« organisation apprenante ».

68 Ainsi, au fil des stages a-t-on pu voir une évolution de nos démarches s'enrichissant aussi des expériences menées par les uns et les autres entre deux occasions de se retrouver ensemble. Par exemple, des difficultés ayant amené l'équipe à innover dans l'urgence ont-elles pu donner naissance à des séquences imprévues mais riches et qui seront réutilisées dans d'autres formations (doc. B), qu'il s'agisse de stages de niveau académique, d'universités d'été ou encore de formations pour des publics complètement différents tels que des élus ou des salariés d'entreprises. 

causes de l'apprenante et l'apprenante à son tour, notamment par les plaisirs qu'elle procure, devient une motivation et une des causes du renouvellement du partenariat, donc de sa durée.

72 L'une des formatrices dit par exemple (doc. A) qu'elle n'a aucun intérêt financier ni professionnel, et voire même plutôt des désagréments vis-à-vis de son institution à pratiquer ce partenariat, mais que c'est l'intérêt de la relation humaine dans une équipe qui l'enrichit qui pour elle entretient le partenariat.

73 La durabilité du partenariat et de l'envie de travailler en partenariat deviennent de ce fait des conséquences de l'apprenante, formant un système en boucle.

\section{Une formulation qui vise à donner du sens}

À ce jour, au travers de cette étude qui s'est voulue très pratique mais aussi éthique, il me semble possible de dire que le partenariat apprenant existe et qu'il a été illustré dans cette recherche-action, très fortement au niveau des personnes et au niveau de l'équipe des formateurs et de façon plus timide en terme d'entrée dans une dynamique de partenariat apprenant pour les institutions.

Le partenariat apprenant, nous l'avons vu, relève d'un état d'esprit, une attitude ou posture partenariale. Il exige des processus à mettre en place mais aussi des procédures à inventer notamment au niveau des institutions pour permettre le malaxage et la diffusion des idées, tout en évitant les écueils de la force des habitudes.

Ma vision des choses à plus long terme est très systémique et évolutive, les institutions pouvant au départ donner un cadre général, un programme autorisant ou favorisant le développement d'un partenariat actif au travers de projets non préétablis (de vrais projets...), ceux-ci étant en retour susceptibles d'agir sur la nature des cadres institutionnels pouvant également interagir à leur niveau. Ainsi, en considérant le système partenarial dans sa globalité, en s'appuyant à la fois sur quelques éléments fixes et stables et en introduisant de la nouveauté, il me semble envisageable de sortir des savoirs établis qui ne sont plus véritablement adaptés à nos situations complexes.

La richesse encore une fois serait dans la diversité des personnes, des équipes, des institutions, et dans leur capacité à articuler leurs actions, à croiser et combiner leurs savoirs et savoir-faire pour entrer dans un savoir agir plus pertinent.

Tout ceci se rattache à des convictions fortes qui sont les miennes, celle de la confiance dans les Hommes, dans leur éducabilité, et qui s'inscrit dans l'amour du monde que nous habitons et que nous pouvons construire sans prendre la société de compétition comme une donnée inéluctable et sans penser que tout est déjà écrit. C'est donc pour moi une formulation qui donne vraiment du sens et de l'espoir à des pratiques. 
C'est par ailleurs un souci de précision et de sauvegarde du mot " partenariat » à la façon dont l'académicien Erik Orsenna (2001, p. 89) le souligne dans son dernier ouvrage : «Il faut faire attention aux mots. Ne pas les répéter à tout bout de champ. Ni les employer à tort et à travers, les uns pour les autres, en racontant des mensonges. Autrement, les mots s'usent. Et parfois il est trop tard pour les sauver. "

Enfin, pour moi il ne s'agit pas d'un hasard si partenariat apprenant et éducation à l'environnement se trouvent reliés dans la mesure où ils vont de pair au travers de leurs pratiques contre deux illusions : celle que l'on habiterait seul le monde et celle que l'on pourrait apprendre seul.

\section{BIBLIOGRAPHIE}

Calame, P. (1996). La pratique de la gouvernance - pour un monde responsable et solidaire au XXIème siècle. Genève : IUED.

Goffin, L. (1998-1999). Pour une recherche en éducation relative à l'environnement « centrée sur l'objet partagé ». Éducation relative à l'environnement : Regards, Recherches, Réflexions, 1, 41-63.

Labelle, J.-M. (1996). La réciprocité éducative. Paris : PUF , Collection Pédagogie d'aujourd'hui.

Mallet, J. (dir.). (1996). L’organisation apprenante (Tomes I et II). Marseille : Université de Provence.

Nonaka, I. et Takeuchi, H. (1997). La connaissance créatrice, la dynamique de l'entreprise apprenante. Bruxelles : De Boeck Université.

Orsenna, E. (2001). La grammaire est une chanson douce. Paris : Stock.

Sauvé. L. (2000). Le partenariat en ERE : pertinence et défis. In Actes du Colloque « Éducation à l'environnement : nouveaux publics, nouveaux partenaires ", coordonné par l'Ifrée, Futuroscope Poitiers, 3 et 4 mai 2000 .

Sauvé, L. (1998-1999). Un patrimoine de recherche en construction. Éducation relative à l'environnement : Regards, Recherches, Réflexions, 1, 13-40.

Schön, D. A. (1994). Le praticien réflexif : à la recherche du savoir caché dans l'agir professionnel. Montréal : Les Éditions Logiques.

Sibony, D. (1991). Entre-deux, l'origine en partage. Paris : Seuil.

\section{RÉSUMÉS}

Cette recherche-action s'appuie sur des formations de formateurs co-organisées par deux institutions relevant de l'Éducation nationale, un réseau associatif et un institut français. Elle explore les pratiques afin de mieux comprendre le fonctionnement et l'efficacité du système partenarial dans son ensemble. L'article présente l'ensemble de la démarche de recherche montrant que le partenariat est "apprenant » dans la mesure où il permet la création de 
connaissances et l'accès à des compétences nouvelles et inattendues, aussi bien au niveau des personnes que des organisations. Il explore des indicateurs de qualité du partenariat et montre la cohérence entre ces pratiques et celles de l'éducation relative à l'environnement (ERE) tout en cherchant à cerner les atouts et limites de cette notion nouvelle de partenariat apprenant.

This research-action is based on trainer's training sessions coorganised by two institutions coming under the Ministry of Education, by a network of associations and by a French institute. It explores the practices in order to get a better understanding of the functioning and of the effenciency of the partnership system as a whole. This paper presents the entire research process and shows that the partnership is "teaching " insofar as it allows the production of knowledge and access to new and unexpected skills, at a personal level as well as at the institutional level. It explores quality indicators of partnership and shows the consistency between these practises and those of environmental education, while trying to define the assets and the limits of this new notion of « teaching " partnership.

\section{AUTEUR}

\section{YANNICK BRUXELLE}

Yannick Bruxelle est professeure agrégée de Sciences de la Vie et de la Terre, en poste au Rectorat de Poitiers notamment pour mettre en place des actions de formation continue visant le développement de projets interdisciplinaires et partenariaux dans le domaine de l'ERE avec des élèves. Engagée pendant trois années dans une recherche avec l'Université de Tours, elle continue cette démarche dans le cadre d'un troisième cycle à l'Université de Rennes 2 (sous la direction de Paul Taylor). Elle est par ailleurs membre active du Réseau national français École et Nature. 